\title{
Headache and cognitive profile in children: a cross-sectional controlled study
}

\author{
Pasquale Parisi - Alberto Verrotti • Maria Chiara Paolino • Antonella Urbano • \\ Mariangela Bernabucci $\cdot$ Rosa Castaldo $\cdot$ Maria Pia Villa
}

Received: 12 August 2009/Accepted: 1 October 2009/Published online: 20 October 2009

(C) Springer-Verlag 2009

\begin{abstract}
We investigated whether children affected by tension-type headache and migraine without aura, compared with a healthy control group that was matched by age, culturally and socioeconomically display a diverse intellectual functioning and have a separate "cognitive profile". A cross-sectional study was conducted from January 2006 to November 2008 at "Sapienza University" in Rome. A total of 134 children were diagnosed as being affected by either migraine without aura (93) or tensiontype headache (41). On the basis of our exclusion/inclusion criteria, we enrolled 82 of these 134 children, 63 of whom were affected by migraine without aura and 19 by tensiontype headache. On entry, cognitive functions were assessed in both the affected subjects and the control group by the Wechsler Intelligence Scale for Children-revised. Significant differences were found between the headache and control groups in the mean total intelligence quotient and verbal intelligence quotient scores $(p<0.001)$. Significant negative correlations were found between the total intelligence quotient, verbal intelligence quotient, performance intelligence quotient and the frequency of attacks $(r=$ -0.55 and $p<0.001, r=-0.61$ and $p<0.001, r=$ -0.29 and $p<0.01$, respectively), as well as between the
\end{abstract}

P. Parisi $(\bowtie) \cdot$ M. C. Paolino - A. Urbano - M. Bernabucci · R. Castaldo - M. P. Villa

Child Neurology, Headache Paediatric Center, Paediatric Sleep

Centre, Chair of Paediatrics, II Faculty of Medicine,

Sapienza University, c/o Sant'Andrea Hospital,

Via di Grottarossa, 1035-1039 Rome, Italy

e-mail: pasquale.parisi@uniroma1.it; parpas@iol.it

A. Verrotti

Child Neurology, Paediatric Department, Chair of Paediatrics,

University of Chieti, Chieti, Italy total intelligence quotient score and the age at headache onset $(r=0.234, p<0.05)$. Our results suggest that the cognitive profile of children affected by headache should be assessed at the first child neurology outpatient observation. From a therapeutic point of view, although within a normal range, the abilities most likely to be less brilliant in such children are verbal skills.

Keywords Headache - Migraine - Cognitive profile . Intellectual performances $\cdot$ Psychometric tests

\section{Introduction}

Headache is a common disorder, largely diffuse in childhood and adolescence. The revised pediatric classification criteria, introduced by the International Headache Society in 2004 [1,2], have led to a more accurate definition of the various forms of headache in childhood and have resulted in a more careful differential diagnosis between migraine, (with $[\mathrm{MA}]$ and without aura $[\mathrm{MoA}]$ ) and tension-type headache (TTH).

Although MA patients [3-6] appear to be the more vulnerable group from a neuropsychological point of view, all migraine patients (i.e., MoA and MA) have been investigated from a cognitive point of view.

Cognitive impairment in tests of perception [3, 7-11], visual attention [12], information speed processing [11-13], simple reaction time (RT) [14] and verbal ability [15-17] have been documented in migraine subjects.

However, not all studies have reported impaired cognitive performance $[18,19]$. There is empirical evidence that patients with migraine, including those with aura, do not have a higher risk of long-term neuropsychological impairment [10, 16, 17]. 
In brief, the few studies available on cognitive dysfunction in pediatric headache have yielded contrasting results. These discrepancies may be due to various reasons, including the fact that a migraine-free control group was not used, the nature of the neuropsychological tests administered was too broad or only a highly specific cognitive function was investigated [12, 14, 17, 20, 21].

As regards the cognitive side effects of therapy, there is evidence that ergotamine long-term prophylaxis may affect cognitive information processing [22] and that processing does not fully return to normal after the therapy has ended.

In view of these contrasting findings and the lack of data in adult and, particularly, in the developmental age, we decided to investigate, by administering the Wechsler Intelligence Scale for Children-revised (WISC-R) in a cross-sectional controlled study, whether children affected by either MoA or TTH who had never taken anti-migraine therapy exhibited different intellectual functioning when compared with a headache-free control group that was matched by age, culturally and socioeconomically. WISC$\mathrm{R}$ is one of the tests used throughout the world to assess the global capacity of children between 6 and 16 years of age, to act purposefully, to think rationally and to deal effectively with their environment.

\section{Patients and methods}

Characteristics of the patients and exclusion criteria

This cross-sectional study was conducted from January 2006 to November 2008 at the Child Neurology Outpatient Service, Paediatric Chair of the Second Faculty of Medicine, "La Sapienza" University in Rome, Italy. In this period, 134 children were diagnosed as being affected by MoA (93 children) and TTH (41 children). In the same period, eight children were diagnosed as being affected by migraine with aura (MA). As only two of these eight children satisfied the inclusion criteria, MA subjects were excluded from the study for statistical reasons.

For the purposes of this study, the following exclusion criteria were applied: (a) presence of any other systemic diseases or major psychiatric disorders; (b) association of different types of headache; (c) anamnesis of previous antimigraine prophylaxis therapy; (d) alterations in the neuroradiological (MRI), neurophysiological (EEG) or blood chemistry test findings; (e) any type of anomaly detected at the neurological examination. The inclusion criteria were: (a) age between 6 and 16 years; (b) diagnosis of primary headache.

According to the exclusion/inclusion criteria and the revised international classification for headache disorders (ICHD-2), 82 subjects (34 males and 48 females, age
$10.9 \pm 2.8 \mathrm{SD}$ years) were enrolled in the study, 63 of whom had MoA (25 males and 38 females, age $11.0 \pm 2.9$ SD years) and 19 TTH (9 males and 10 females, age $10.9 \pm 2.6$ years). None of the patients took any prophylactic medication before they underwent the cognitive assessment by means of the WISC-R, according to the study design. The frequency of the attacks, the duration of the patient's history of headache and the age at onset were also recorded.

The control group, which was matched for age, sex, body mass index (BMI), racial and socioeconomic status, family education background and time missed at school, was composed of 79 healthy children (27 males and 52 females), attending a state school in the same geographic area. All the children were screened for any pathological condition potentially related to cognitive impairment (respiratory diseases, asthma, allergy, sleep disorders, epilepsy, ADHD, obesity, family history of mental retardation or any other neuropsychiatric conditions).

\section{Cognitive assessment}

Cognitive functions were analyzed by means of the WISC$\mathrm{R}$, an intelligence test validated for children between the ages of 6 and 16 years. The test comprises ten core subtests and two supplemental tests. These subtests generate a fullscale score (FSI, or total intelligence quotient TIQ), and two composite scores known as indices : the verbal comprehension score (VIQ) (including vocabulary, similarities, comprehension, information, arithmetic, and digit span as the supplemental test) and performance score (PIQ) (including block design, picture stories, picture completion, puzzles, coding and mazes as the supplemental test). The supplemental subtests are used to accommodate children in rare cases, or to compensate for results affected by interruptions or other circumstances. The cognitive assessment was performed after informed consent was obtained from the parents of all the children enrolled. The study was approved by the local ethics committee of the "La Sapienza" University of Rome.

Administration of the WISC-R (which usually requires 75-80 $\mathrm{min}$ ) took place on a symptom-free day at least 2 days after the last headache attack to rule out the possibility of the subjects being in a postdrome period and, consequently, of any effects of the prodrome state on their neuropsychological performance. We did not have to use the supplemental subtests. According to the study design, all the patients underwent magnetic resonance imaging (MRI) of the brain, which yielded negative results in all enrolled headache children, a set of blood chemistry analyses and a comprehensive neurological examination, which were normal too. The EEG was normal in all the children enrolled. 
Statistical analysis

Data are expressed as the mean $\pm \mathrm{SD}$ (standard deviation). Unpaired $t$ test or ANOVA with post hoc Scheffe test were used for comparisons between two or more subgroups as required. Pearson coefficient correlations between variables were determined. Significant correlated IQ score variables and selected clinical and anamnestic variables were then included in a stepwise linear multiple regression model using TIQ, VIQ and PIQ separately as dependent variables and the headache subtypes, frequency of the attacks, the duration of the patient's history of headache and the age at onset as independent variables. $P$ values of less than 0.05 were considered to be statistically significant. Statistical analysis was performed using a commercial software package (SPSS, version 11; SPSS; Chicago, IL).

\section{Results}

Table 1 shows the clinical and anthropometric parameters of all the groups: A (control group composed of 79 matched, healthy children), B (MoA, composed of 63 children) and $\mathrm{C}$ (TTH, composed of 19 children). The ANOVA test with post hoc Scheffe is also shown in Table 1.

There were significant differences in the mean TIQ and VIQ scores between the groups $(108.1 \pm 13.2$ in the MoA group and $110.6 \pm 15.7$ in the TTH group vs. $115.8 \pm$ 10.6 in healthy children, $p<0.001$, for the TIQ score and, respectively, $108 \pm 14.8$ and $108.9 \pm 15.6$ vs. $118.2 \pm$ $11.8, p<0.001$, for the VIQ score). The MoA group scored lowest in both the TIQ and VIQ. There were no significant differences in the PIQ score between the three groups (Table 2).

The analysis of each subset item revealed significant differences between MoA patients and healthy children in four verbal items and two performance items, whereas the
TTH group differed from healthy children in only one verbal item (Table 2). Significant negative correlations were found between the TIQ, VIQ, PIQ and the frequency of attacks $(r=-0.55$ and $p<0.001, r=-0.61$ and $p<0.001, r=-0.29$ and $p<0.01$, respectively), as shown in Figs. 1a, b and 2a. A significant correlation was found between the age at headache onset and the TIQ $(r=0.234, p<0.05)$ (Fig. 2b).

Stepwise linear multiple regression, investigating each of the correlated WISC-R variables (TIQ, VIQ, PIQ) separately as dependent variables and the headache subtypes, frequency of the attacks, the duration of the patient's history of headache and the age at onset as independent variables, was performed. The results show that the frequency of attacks was an independent predictor of alteration for all the dependent variables considered, and that age at headache onset was an independent predictor of alteration for the TIQ.

\section{Discussion}

Few studies designed to assess the impact of headache on neurocognitive performance in pediatric age have been conducted. In one such study [20], based on a cohort of 20 children with migraine, aged 7-11 years, intelligence, digit span and visual-motor integration were normal, whereas performance in short- and long-delayed memory tasks was significantly impaired. Another study, in which children aged 6-12 years with migraine were compared with their healthy siblings, did not reveal any significant differences on a scale assessing sequential and simultaneous information processing [21].

A longitudinal study conducted on migraineurs from the age of 3 to 26 years [17] reported impaired verbal skills in migraineurs compared with the control group; cognitive impairment was more evident during infancy and

Table 1 Anthropometric and clinical characteristics

\begin{tabular}{|c|c|c|c|c|c|c|c|c|c|c|}
\hline & \multicolumn{2}{|c|}{$\begin{array}{l}\text { Group A (control } \\
\text { group) }(n=79)\end{array}$} & \multicolumn{2}{|c|}{$\begin{array}{l}\text { Group B MoA } \\
\text { children }(n=63)\end{array}$} & \multicolumn{2}{|c|}{$\begin{array}{l}\text { Group C TTH } \\
\text { children }(n=19)\end{array}$} & \multirow[t]{2}{*}{ Anova $p<$} & \multicolumn{3}{|c|}{ Sheffe $p<$} \\
\hline & Mean & SD & Mean & SD & Mean & SD & & 0 vs. 1 & 0 vs. 2 & 1 vs. 2 \\
\hline Age (years) & 10.04 & 2.4 & 11.0 & 2.9 & 10.8 & 2.7 & NS & NS & NS & NS \\
\hline Centile BMI $\left(\mathrm{kg} / \mathrm{m}^{2}\right)$ & 60.4 & 6.8 & 64.3 & 6.2 & 59.1 & 9.4 & NS & NS & NS & NS \\
\hline Duration of disease (years) & - & - & 2.2 & 2.1 & 2.3 & 1.8 & NS & - & - & - \\
\hline Age at onset & - & - & 9.2 & 6.4 & 8.6 & 3.4 & NS & - & - & - \\
\hline Frequency of the attacks (events/month) & - & - & 9.7 & 5.4 & 9.8 & 5.7 & NS & - & - & - \\
\hline$n$ & & $n$ & & & $n$ & & & & & $\chi^{2}(p<)$ \\
\hline Sex (male) & 4.2 & 25 & & & 9 & & 7.4 & & & NS \\
\hline
\end{tabular}


Table 2 Scores at neurocognitive assessment by WISC-R

\begin{tabular}{|c|c|c|c|c|c|c|c|c|c|c|}
\hline \multirow[t]{2}{*}{ WISC-R variables } & \multicolumn{2}{|c|}{$\begin{array}{l}\text { Group A } \\
\text { (control group) }(n=79)\end{array}$} & \multicolumn{2}{|c|}{$\begin{array}{l}\text { Group B } \\
\text { MoA children }(n=63)\end{array}$} & \multicolumn{2}{|c|}{$\begin{array}{l}\text { Group C TTH } \\
\text { children }(n=19)\end{array}$} & \multirow[t]{2}{*}{ ANOVA $p$} & \multicolumn{3}{|c|}{ Sheffe $p$} \\
\hline & Mean & SD & Mean & SD & Mean & SD & & 0 vs. 1 & 0 vs. 2 & 1 vs. 2 \\
\hline TIQ & 115.8 & 10.6 & 108.1 & 13.2 & 110.6 & 15.7 & $<0.001$ & $<0.001$ & NS & NS \\
\hline VIQ & 118.2 & 11.8 & 108 & 14.8 & 108.9 & 15.6 & $<0.000$ & $<0.000$ & $<0.02$ & NS \\
\hline PIQ & 110.1 & 11.2 & 106.1 & 13.8 & 110 & 14.9 & NS & NS & NS & NS \\
\hline Information & 11.5 & 2.6 & 9.5 & 2.8 & 9.9 & 3.2 & $<0.000$ & $<0.000$ & NS & NS \\
\hline Similarities & 13.1 & 3 & 11.5 & 3.4 & 11.5 & 4.1 & $<0.01$ & $<0.02$ & NS & NS \\
\hline Arithmetic & 12.1 & 2.5 & 11.2 & 3.2 & 11 & 2.1 & NS & NS & NS & NS \\
\hline Vocabulary & 15.5 & 2.2 & 14 & 3.3 & 14.3 & 4.3 & $<0.01$ & $<0.02$ & NS & NS \\
\hline Comprehension & 12.4 & 2.4 & 10.4 & 2.8 & 9.5 & 2.5 & $<0.000$ & $<0.000$ & $<0.000$ & NS \\
\hline Picture completion & 11.6 & 2.6 & 9.9 & 2.9 & 10.2 & 2.5 & $<0.001$ & $<0.001$ & NS & NS \\
\hline Picture stories & 10.9 & 2.4 & 11.7 & 2.5 & 11.8 & 3.3 & NS & NS & NS & NS \\
\hline Block design & 11.2 & 2.3 & 11.2 & 2.9 & 11.5 & 3.2 & NS & NS & NS & NS \\
\hline Puzzles & 11.9 & 2.6 & 11.4 & 2.8 & 11.6 & 2.8 & NS & NS & NS & NS \\
\hline Coding & 11.9 & 2.4 & 10.6 & 3.6 & 10.9 & 2.2 & $<0.02$ & $<0.03$ & NS & NS \\
\hline
\end{tabular}

adolescence, i.e., from 3 to 13 years of age. However, the subtle verbal deficits exhibited by migraineurs may have affected later achievement, as suggested by the fact that high school grades and examination scores were significantly lower in migraineurs than in the control group, and that fewer migraineurs achieved secondary school qualifications or degrees.

A recent study [14] reported a significant dysfunction in the information processing rate alone. The simple reaction time (RT) to visual stimuli was slow in a significantly larger number of migraineurs than in the normal population; moreover, the RT was the only variable that correlated significantly with the frequency of headache attacks. The authors of that study suggested that there may be a slowdown in information processing within the posterior cortical areas responsible for detecting the visual stimulus, and within the premotor areas that program and implement the motor response. However, a serious limitation of that study was the lack of a matched, headache-free control group.

Yet another study [12] was conducted more recently on 30 children with migraine and 30 healthy children (control group). Attention can be defined as the capacity to respond to significant stimuli to the detriment of others. Attention is a complex, specific neurological function that is highly dependent on a set of anatomical structures such as the brainstem, cerebral cortex and limbic system. Children with migraine in that study exhibited an impairment in all the variables except the RT in the visual attention test tasks, when compared with controls. Although the attention task performance fell within the normal range in both groups, the migraine group had difficulties in selective attention and, in particular, in alternate attention. The authors did not find a correlation between the duration of migraine or the attack frequency and the attention impairment in the migraine group.

Our results revealed a statistically significant difference between children affected by TTH and the control group with regard to the TIQ score (full intelligence quotient scale); this difference was even more significant between children affected by MoA and the control group (Table 2).

There were no significant differences between the groups in the performance intelligence scale quotient (PIQ) score, whereas statistically significant differences emerged in the verbal intelligence scale quotient (VIQ) score between both headache groups and the control group, with a markedly higher statistical difference $(p<0.000)$ (Table 2) again being documented between MoA patients and the control group.

In accordance with a previous report [17], our patients exhibited a particular significant differences in verbal skills. Waldie et al. [17] stated that verbal performance was not significantly influenced by migraine attacks "per se", but was due rather to a "not-identified" prenatal shared risk factor, thereby suggesting that the origins of both migraine headache and cognitive impairment are to be found in a very early developmental phase. In addition, they found more marked cognitive impairment during infancy and adolescence, from 3 to 13 years of age. Our results support this hypothesis, though only to some extent. Indeed, in our patients, linear multiple regression models (Table 3; Figs. 1, 2) showed that the frequency of the attacks were independent predictors of alteration for all the dependent variables considered (TIQ, VIQ, PIQ) and that age at 
(a)

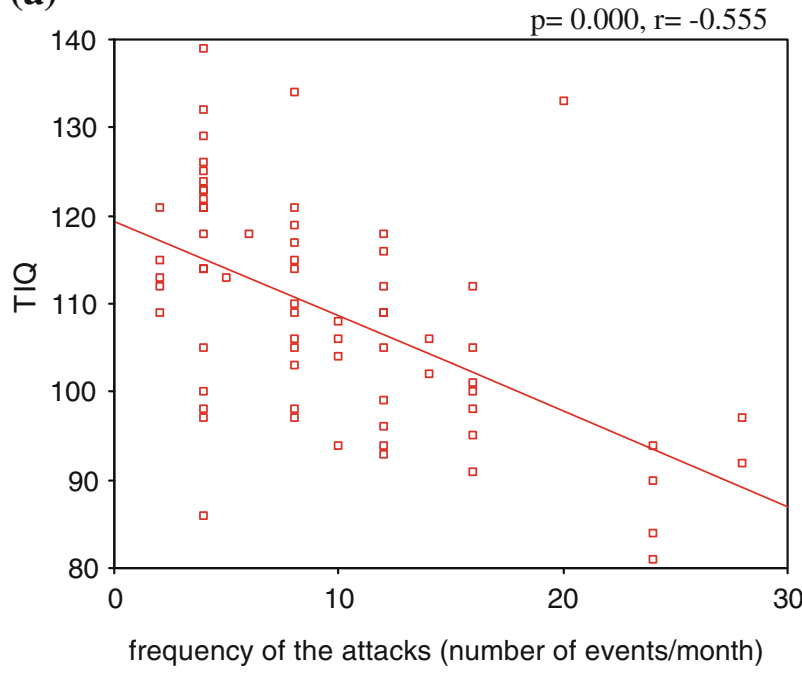

(b)

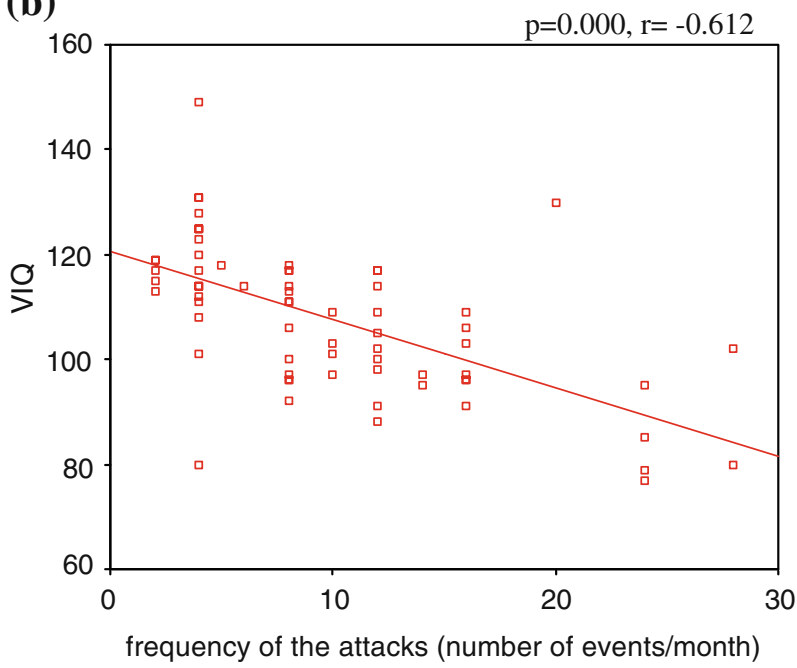

Fig. 1 a Correlation between total intelligence quotient (TIQ) and the frequency of the attacks (number of events/month) in all headache patients. b Correlation between verbal intelligence quotient (VIQ) and the frequency of the attacks (number of events/month) in all headache patients

headache onset was an independent predictor of alteration for TIQ. Our findings do not thus confirm that the migraine headaches and cognitive impairment arise in an early developmental phase. On the contrary, our results suggest that early age at onset and a high frequency of headache attacks are associated with cognitive impairment. In other words, cognitive impairment in headache is, according to our data, exacerbated by age at onset and the frequency of attacks, probably owing to the immaturity of the central nervous system in the developmental age. Actually, it would be interesting to study if a separate subcategory (subjects with more than 15 days of TTH or MoA per months, the so-called chronic MoA or TTH forms) would
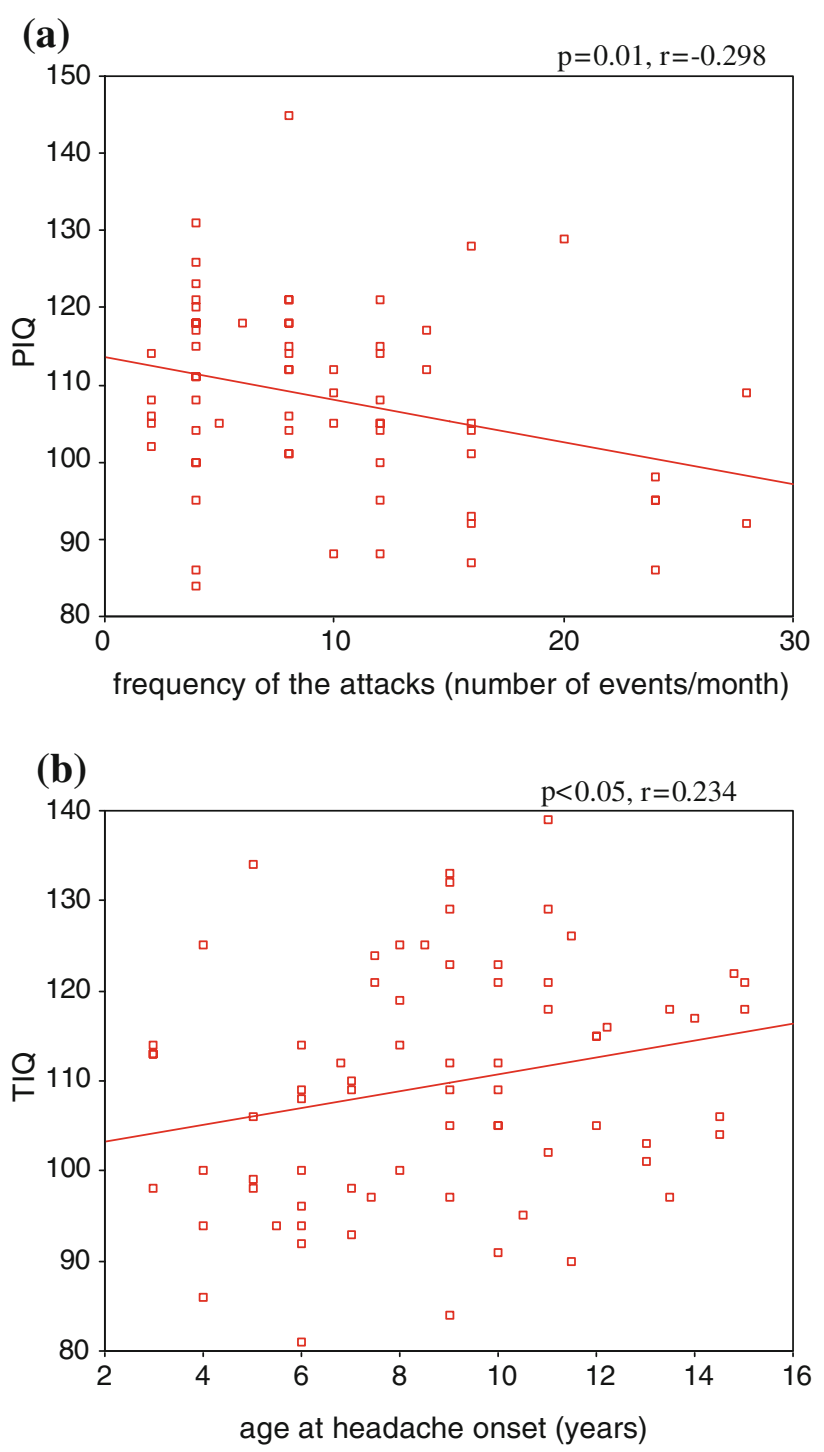

Fig. 2 a Correlation between performance intelligence quotient (PIQ) and the frequency of the attacks (number of events/month) in all headache patients. b Correlation between total intelligence quotient (TIQ) and the age at headache onset (years) in all headache patients

have maintained a statistical significance (Table 3; Figs. 1, 2), but unfortunately this type of statistical analysis was not performed due to the small sample available in this subcategory.

As we had conducted a cross-sectional controlled study, we had no data on cognitive long-term prognosis or academic achievement in adult age and we could not investigate the possibility of reversing and improving cognitive performances by means of behavioral/cognitive or pharmacological therapies, though the latter hypothesis had previously been supported by promising results [23].

However, we do confirm the prevalence of statistical differences in the verbal comprehension subscale, as 
Table 3 Stepwise linear multiple regression models investigating TIQ, VIQ and PIQ separately as dependent variables, and the headache subtypes, frequency of the attacks, the duration of the patient's history and the age at onset as independent variables

\begin{tabular}{|c|c|c|c|c|c|c|}
\hline \multirow[t]{3}{*}{ Independent variables } & \multicolumn{6}{|c|}{ Dependent variables } \\
\hline & \multicolumn{2}{|l|}{ TIQ } & \multicolumn{2}{|l|}{ VIQ } & \multicolumn{2}{|l|}{ PIQ } \\
\hline & $\begin{array}{l}\text { Standardized } \\
\text { coefficients beta }\end{array}$ & $p$ & $\begin{array}{l}\text { Standardized } \\
\text { coefficients beta }\end{array}$ & $p$ & $\begin{array}{l}\text { Standardized } \\
\text { coefficients beta }\end{array}$ & $p$ \\
\hline Frequency (events/month) & -0.551 & 0.000 & -0.607 & 0.000 & -0.301 & 0.012 \\
\hline Age at headache onset & -0.069 & 0.05 & 0.207 & 0.065 & 0.177 & 0.190 \\
\hline Duration of disease & 0.224 & 0.548 & -0.053 & 0.634 & -0.061 & 0.649 \\
\hline Headache subtypes & 0.088 & 0.380 & 0.028 & 0.770 & 0.145 & 0.220 \\
\hline
\end{tabular}

previously reported [17], even though all the other verbal subtest (vocabulary, similarities, information) scores, except the arithmetic subtest, were significantly lower in our MoA patients than in the control group. In children affected by TTH, the verbal comprehension subtest score alone was significantly lower than in the control group.

The verbal comprehension subtest investigates individual skills regarding the management of social conditions or common concepts according to the criteria of appropriateness and common sense, using social judgment.

It is not clear why the verbal comprehension subscale appears to be more clearly involved in such patients than other verbal skills, or why the verbal subscales are all involved and the performance subscales are not. It should, however, be borne in mind that linguistic skills are required to perform all verbal subscale items, both on the receptive and expressive sides. Also, language is a relatively recent phylogenetic acquisition and is the most complex and peculiar ability in the human being's evolution. In all the psychometric standardized tests used to assess the intelligence quotient (IQ) in children, such as the WISC-R and Wechsler Preschool and Primary Scale of Intelligence (WPPSI), language ability scores are closely correlated with the global IQ scores.

On the other hand, there is no universally accepted definition of what we mean by "intelligence". We know that intelligence is not topographically correlated with a specific cortical area. On the contrary, numerous cortical and subcortical neuronal networks appear to cooperate synergistically in cognitive performances [24]. In brief, intelligence is believed to be a widespread brain function that is not topographically identifiable.

In headache patients, and particularly in those affected by migraine, an involvement of cognitive function might be related (functionally [25] or structurally [26]) to cortical areas, such as the frontal and prefrontal areas, as a consequence of poor sleep [25], and sub-cortical areas, as a consequence of iron accumulation in deep brain nuclei (as documented in adult age [26]). It has recently been reported that the higher cognitive functions express the activity of both cortical and subcortical diffuse neuronal networks [24]. According to the "diffuse hypothesis", and bearing in mind the most recent theory in the pathophysiology of migraine (trigeminovascular theory and cortical spreading depression) [27, 28], we may postulate that repeated activation of several cortical or subcortical neuronal networks during migraine attacks (or the "pain phase" in general) is associated with the involvement (functional or structural) [25, 26] of these central nervous system structures. This chronic, recurrent hyperactivation might in turn result in cognitive involvement.

It would be intriguing to investigate, first, whether early recognition and a correct and prompt anti-headache therapy can prevent this cognitive different profile and, second, whether the cognitive profile associated with headache is reversible at any time during the course of the disease. Lastly, any cognitive therapy will have to take into consideration the peculiar needs of verbal skill involvement, which is, as our findings confirm, the prevalent type of skills whose statistical lower score is associated in a significant way with headache [17].

We would like to conclude with some reflections on the weak points in our investigation. One limitation of our study was the lack of a control group with pain other than migraine/headache, and another might be the type of study design. Indeed, although we enrolled the patients in our study groups prospectively and consecutively, the design we adopted was a controlled cross-sectional study, which is considered to be weaker than a prospective longitudinal controlled study.

The last, but not the least, important weak point in our investigation was that our "control group" attained very good scores (at the highest limit of the average) and so we could not define MA or TTH group as an "impaired group", because all the groups (MA, TTH and control group) scored in the "normal range". Nonetheless, the statistical significant differences among the three groups were clearly documented (see Tables and Figures).

Speaking about other Italian children (the "brilliant" control group), another cognitive study from Italy using the 
WISC (investigating hypothyroidism and not headache) [29] reported an average WISC score of 110. In any case, for this reason we have to stress that this clinical data cannot be transferred to the general population.

Finally, in our opinion, the most relevant data from our study is that the only significant independent variable for cognitive performances was the frequency of attacks (Table 3; Figs. 1, 2). This is a crucial finding, which suggests the need for further studies addressing the effects of an early intervention in the pediatric age [30].

Conflict of interest None.

\section{References}

1. Winner P, Martinez W, Mante L, Bello L (1995) Classification of paediatric migraine: proposed revisions to the IHS criteria. Headache 35:407-410

2. Headache Classification Committee of the International Headache Society (2004) The international classification of headache disorders, 2nd edn. Cephalalgia 24(Suppl 1):1-160

3. Hooker WD, Raskin NH (1986) Neuropsychologic alterations in classic and common migraine. Arch Neurol 43:709-812

4. Chronicle EP, Wilkins AJ, Coleston DM (1995) Thresholds for detection of a target against background grating suggest visual dysfunction in migraine with aura but not migraine without aura. Cephalalgia 15:117-122

5. Chronicle E, Mulleners W (1994) Might migraine damage the brain? Cephalalgia 14:415-418

6. Mulder EJCM, Linssen WHJP, Passchier J, Orlebeke JF, de Geus EJC (1999) Interictal and postictal cognitive changes in migraine. Cephalalgia 19:557-564

7. Ardila A, Sanchez E (1988) Neuropsychologic symptoms in the migraine syndrome. Cephalalgia 8:67-70

8. Ai Q (1992) A neuropsychologic study in migraine patients. Zhonghua Shen Jing Jing Shen Ke Za Zhi 25:92-95

9. Scherer P, Bauer H, Baum K (1997) Alternate finger tapping test in patients with migraine. Acta Neurol Scand 96:392-396

10. Le Pira F, Zappala G, Giuffrida S, Lo Bartolo ML, Morana R, Lanaia F (2000) Memory disturbances in migraine with and without aura: a strategy problem? Cephalalgia 20:475-478

11. Calandre EP, Bembibre J, Arnedo ML, Becera D (2002) Cognitive disturbances and regional cerebral blood flow abnormalities in migraine patients: their relationship with the clinical manifestations of the illness. Cephalalgia 22:291-302

12. Villa TR, Correa Moutran AR, Sobirai Diaz LA, Pereira Pinto MM, Carvalho FA, Gabbai AA, de Souza Carvalho D (2009) Visual attention in children with migraine: a controlled comparative study. Cephalalgia [Epub ahead of print]. doi:10.1111/ j.1468-2982.2008.01767.x

13. Zeitlin C, Oddy M (1984) Cognitive impairment in patients with severe migraine. Br J Clin Psychol 23:27-35

14. Riva D, Aggio F, Vago C, Nichelli F, Andreucci E, Paruta N, D'Arrigo S, Pantaleoni C, Bulgheroni S (2006) Cognitive and behavioural effects of migraine in childhood and adolescence. Cephalalgia 26:596-603
15. Sinforiani E, Farina S, Mancuso A, Manzoni CG, Bono G, Mazzocchi A (1987) Analysis of higher nervous functions in migraine and cluster headache. Funct Neurol 2:69-77

16. Leijdekkers MLA, Passchier J, Goudswaard P, Menges LJ, Orlebeke JF (1990) Migraine patients cognitively impaired? Headache 30:352-358

17. Waldie KE, Hausmann M, Milne BJ, Poulton R (2002) Migraine and cognitive function. A life-course study. Neurology 59:904-908

18. Bell BD, Primeau M, Sweet JJ, Lofland KR (1999) Neuropsychological functioning in migraine in headache, non-headache chronic pain, and mild traumatic brain injury patients. Arch Clin Neuropsychol 14:389-399

19. Sandrini G, Friberg L, Jänig W, Jensen R, Russell D, Sanchez del Rìo M, Sand T, Schoenen J, Buchem M, van Dijk JG (2004) Neurophysiological tests and neuroimaging procedures in nonacute headache: guidelines and recommendations. Eur J Neurol 11:217-224

20. D'Andrea G, Nertempi P, Ferro Milone F, Joseph R, Cananzi JR (1989) Personality and memory in childhood migraine. Cephalalgia 9:25-28

21. Haverkamp F, Honscheid A, Muller-Sinik K (2002) Cognitive development in children with migraine and their unaffected siblings. Headache 42:776-779

22. Evers S, Schmidt F, Bauer B, Voss H, Grotemeyer K-H, Husstedt IW (1999) The impact of ergotamine-induced headache and ergotamine withdrawal on information processing. Psychopharmacology 142:61-67

23. Rains JC (2008) Change mechanisms in EMG biofeedback training: cognitive changes underlying improvements in tension headache. Headache 48:735-737

24. Münte TF, Heldmann M, Hinrichs H, Marco-Pallares J, Krämer UM, Sturm V, Heinze HJ (2008) Contribution of subcortical structures to cognition assessed with invasive electrophysiology in humans. Front Neurosci 2:72-78

25. Seidel S, Hartl T, Weber M, Matterey S, Paul A, Riederer F, Gharabaghi M, Wöber-Bingöl C, Wöber C; the PAMINA Study Group (2009) Quality of sleep, fatigue and daytime sleepiness in migraine-a controlled study. Cephalalgia [Epub ahead of print]. doi:10.1111/j.1468-2982.2008.01784.x

26. Kruit MC, Launer LJ, Overbosch J, van Buchem MA, Ferrari MD (2008) Iron accumulation in deep brain nuclei in migraine: a population-based magnetic resonance imaging study. Cephalalgia 29:351-359

27. Moskowitz MA, Nozaki K, Kraig RP (1993) Neocortical spreading depression provokes the expression of C-fos proteinlike immunoreactivity within trigeminal nucleus caudalis via trigeminovascular mechanisms. J Neurosci 13:1167-1177

28. Ayata C, Jin H, Kudo C, Dalkara T, Moskowitz MA (2006) Suppression of cortical spreading depression in migraine prophylaxis. Ann Neurol 59:652-661

29. Weber G, Mora S, Prina Cerai LM, Siragusa V, Colombini J, Medaglini S, Fornara C, Locatelli T, Comi G, Chiumello G (2000) Cognitive function and neurophysiological evaluation in early-treated hypothyroid children. Neurol Sci 21:307-314

30. Charles JA, Peterlin BL, Rapoport AM, Linder SL, Kabbouche MA, Sheftell FD (2009) Favorable outcome of early treatment of new-onset child and adolescent migraine: implications for disease modification. J Headache Pain 10:227-233 